\title{
Feature Extraction and Evaluation for Classification Models of Injurious Falls Based on Surface Electromyography
}

\author{
Kitaek Lim, PT, BPT, Woochol Joseph Choi, PT, PhD \\ Injury Prevention and Biomechanics Laboratory, Department of Physical Therapy, Yonsei University, Wonju, Korea
}

\author{
Article Info \\ Received February 15, 2021 \\ Revised April 7, 2021 \\ Accepted April 9, 2021 \\ Corresponding Author \\ Woochol Joseph Choi \\ E-mail: wcjchoi@yonsei.ac.kr \\ https://orcid.org/0000-0002-6623-3806
}

\section{Key Words \\ Classification \\ Datamining}

Electromyography

Falls

Injurious falls

Muscle activation
Background: Only $2 \%$ of falls in older adults result in serious injuries (i.e., hip fracture). Therefore, it is important to differentiate injurious versus non-injurious falls, which is critical to develop effective interventions for injury prevention.

Objects: The purpose of this study was to a. extract the best features of surface electromyography (sEMG) for classification of injurious falls, and $b$. find a best model provided by data mining techniques using the extracted features.

Methods: Twenty young adults self-initiated falls and landed sideways. Falling trials were consisted of three initial fall directions (forward, sideways, or backward) and three knee positions at the time of hip impact (the impacting-side knee contacted the other knee ("knee together") or the mat ("knee on mat"), or neither the other knee nor the mat was contacted by the impacting-side knee ("free knee"). Falls involved "backward initial fall direction" or "free knee" were defined as "injurious falls" as suggested from previous studies. Nine features were extracted from sEMG signals of four hip muscles during a fall, including integral of absolute value (IAV), Wilson amplitude (WAMP), zero crossing (ZC), number of turns (NT), mean of amplitude (MA), root mean square (RMS), average amplitude change (AAC), difference absolute standard deviation value (DASDV). The decision tree and support vector machine (SVM) were used to classify the injurious falls.

Results: For the initial fall direction, accuracy of the best model (SVM with a DASDV) was $48 \%$. For the knee position, accuracy of the best model (SVM with an AAC) was $49 \%$. Furthermore, there was no model that has sensitivity and specificity of $80 \%$ or greater.

Conclusion: Our results suggest that the classification model built upon the sEMG features of the four hip muscles are not effective to classify injurious falls. Future studies should consider other data mining techniques with different muscles.

\section{INTRODUCTION}

Osteoporotic hip fractures are common in individuals aged over 65 , and $90 \%$ of them are caused by falls [1]. Furthermore, $20 \%$ of hospitalized older adults die within a year, and $50 \%$ of them suffer from permanent disabilities [2,3]. However, only $1 \%-2 \%$ of falls end up with hip fractures, and this suggests there exist factors that differentiate injurious versus non-injurious falls [4]. If injurious falls are characterized and classified, effective interventions (i.e., exercise programs) could be developed for fall-related hip fractures in older adults.

A risk of hip fracture during a fall is defined as a ratio of the applied force to the fracture strength of the femur. When the applied force becomes greater than the femoral strength during impact, the fall becomes injurious to cause a hip fracture, and several factors have been suggested to determine the injurious falls. Researchers have shown that the hip fracture risk is affected by knee positions at the time of hip impact, and hip impact force becomes 59\% greater when the contactingside knee is free of constraint (i.e., not touching the floor and the contralateral knee) [5,6]. Furthermore, the hip fracture risk increases ten-fold when the fall initiated backward and landed sideways [7].

Efforts have been made to classify and characterize falls using sensors and machine learning algorisms [8,9]. Different sensors like accelerometer, surface electromyography (sEMG), 
pressure sensors and inclinometer have been used, but the accelerometer is the most common due to its convenience and versatility (i.e., light, small and easy to wear during movements) [10-12]. However, the acceleration data are limited to differentiate active versus passive movements, and Roy et al. [13] showed its limitation in distinguishing motion of riding a stationary bike and sitting still. In contrast, sEMG can distinguish active and passive movements, and used for gesture recognition, gait analysis, and limb prosthetic control [14-16]. Furthermore, Xi et al. [17] reported $98.7 \%$ of sensitivity and 98.6\% of specificity in classifying activities of daily living and falls using sEMG.

sEMG collects electrical signals when muscles develop forces [18], and useful information may be extracted from the sEMG signals in time, frequency, or time-frequency domain [19]. However, the frequency and time-frequency domain are not suitable to a real-time pattern recognition due to the complexity of computation [20]. In contrast, sEMG data in time domain can be used easily and fast because no additional processes (i.e., transformation) are required, and the following extracted features are suggested in literature: Average Amplitude Change (AAC) [15], Difference Absolute Standard Deviation (DASDV) [15,21], Integral of Absolute Value (IAV) [9,22,23], Mean Am- plitude (MA) [23,24], Number of Turns (NT) [23], Root mean square (RMS) [21,24], Variance (VAR) [9,22,25], Wilson Amplitude (WAMP) [22], Zero crossing (ZC) [23,26]. These features have been used in the field of pattern recognition (i.e., controlling of a prosthetic upper extremity, daily activity monitoring and fall detection) [9,15,21-24,26].

Selection of appropriate data mining techniques in pattern recognition is the most important to increase the model accuracy. Xi et al. [17] have used Gaussian kernel support vector machine (also called as a radial basis function support vector machine) to classify 8 activities including 7 daily activities and 1 fall, and showed 96.4\% of model accuracy. Keleş and Subaş1 [27] have used a decision tree algorithm to classify muscle activity of individuals with myopathy and neuropathy, and showed $99.3 \%$ of model accuracy.

Against the background, we first extracted features from sEMG of four hip muscles during a fall, and then developed and evaluated models to classify injurious falls using the decision tree and support vector machine (SVM) algorithms based on the extracted features. We hypothesized that the accuracy of our created model would be greater than $80 \%$.

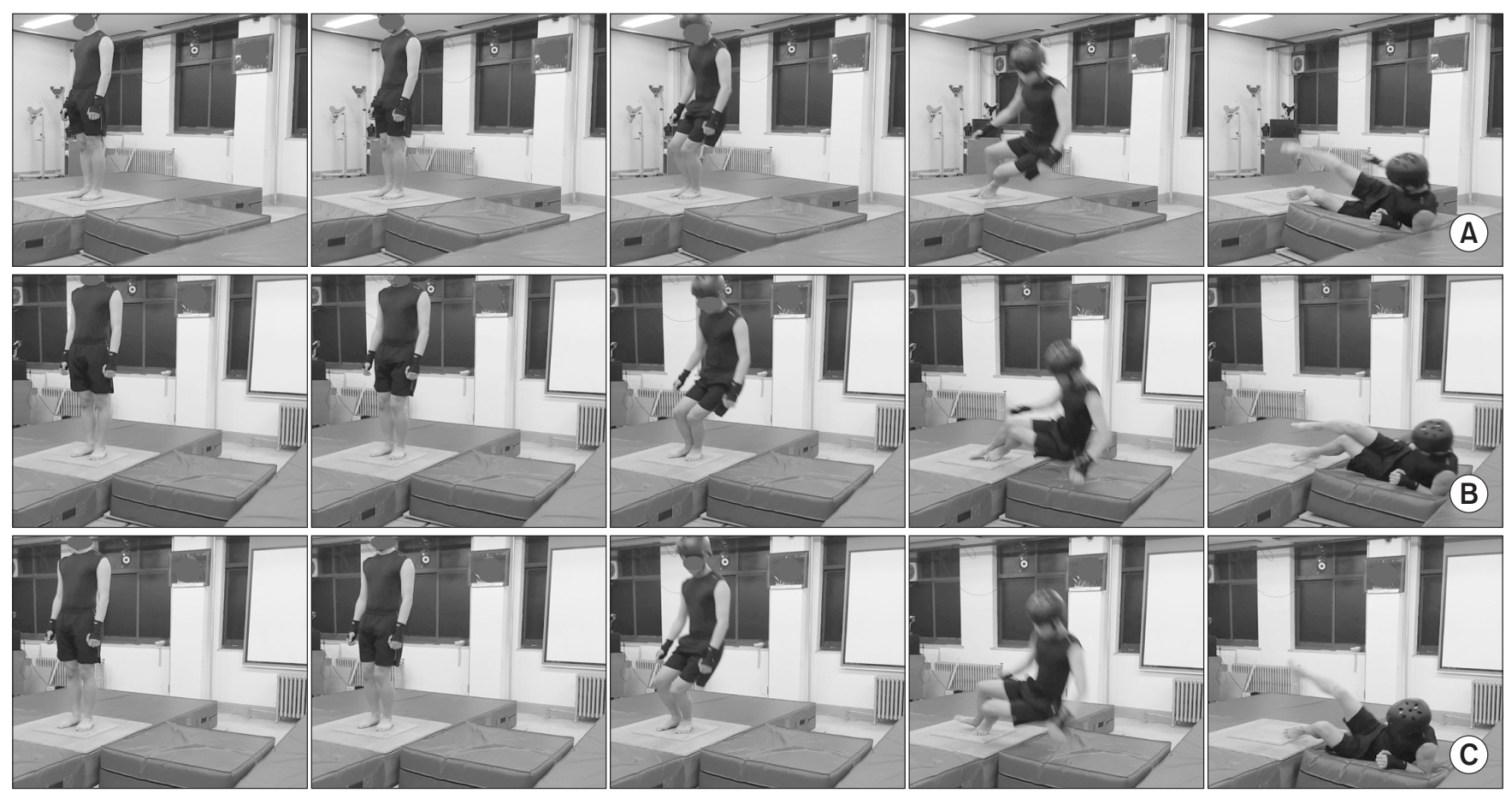

Figure 1. Sample snapshots of falls initiated sideways and landed sideways in a particular participant, where (A) the impacting-side knee contacted the mat, (B) the impacting-side knee contacted the contralateral knee, and (C) neither the impacting-knee nor the mat contacted the impacting-side knee at the time of hip impact during a fall. 


\section{MATERIALS AND METHODS}

\section{Participants and Procedure}

Lower extremity sEMG data published in our companion paper [28] were used as secondary data for this study. While the companion paper reported results of eighteen samples, we included all twenty individuals (10 males and 10 females). Participants' body weight and height averaged $64.9 \pm 11.7 \mathrm{~kg}$ and $166.8 \pm 7.3 \mathrm{~cm}$, respectively. Participants self-initiated falls and landed sideways. Falling trials were acquired with three knee positions at the time of hip impact (the impacting-side knee contacted the mat ("knee on mat") or the contralateral knee ("knee together"), or neither the mat nor the contralateral knee contacted the impacting-side knee ("free knee"), and with three initial fall directions (forward, backward, or sideways) (total falling condition: three knee positions X three initial fall directions) (Figure 1). Each participant fell three times for each falling condition. During falls, activity of the iliopsoas, gluteus maximus and medius, and adductor longus were recorded from the EMG electrodes placed on the belly of the muscles. In this study, injurious falls were defined as falls involved "free knee", or "backward initial fall direction" based on two previous studies - Lim and Choi [6] and Yang et al. [7], respectively.

\section{EMG Data Preparation and Feature Extraction}

The sampling rate of sEMG signals was $3,000 \mathrm{~Hz}$ and the collected EMG signals were filtered with $10 \mathrm{~Hz}$ of high-pass filter and 1,000 Hz of low-pass filter. Time to onset was set as follows:

Time to onset $\mathrm{i}($ frame $)=$

$$
\left.2 \times \sum_{k=1}^{300} R M S \text { (resting state }\right) \leq \sum_{i}^{i+300} R M S_{i}
$$

(where, RMS = Root mean square value of raw signal during a fall with time window $50 \mathrm{msec}$ )

The first 600 milliseconds (1,800 frames) from the onset time were used for the feature extraction. Nine features of the time domain of the sEMG signal were extracted to generate the input variables to be used in fall classification, and the features were described in Table 1. Abbreviations used for each feature are as follows: ( $\mathrm{N}$ = number of the sample, I = i-th sEMG signal, $\mathrm{u}(\mathrm{x})=$ unit step function, $\mathrm{T}=$ threshold). All sEMG features were computed by using Matlab routines (Matlab R2019b; MathWorks Inc., Natick, MA, USA).
Table 1. Abbreviation of extracted feature from the signal of EMG

\begin{tabular}{ll}
\hline \multicolumn{1}{c}{ Extracted feature } & Acronym \\
\hline Integral of absolute value & IAV \\
Variance & VAR \\
Wilson amplitude & WAMP \\
Zero crossing & ZC \\
Number of turns & NT \\
Mean of amplitude & MA \\
Root mean square & RMS \\
Average amplitude change & AAC \\
Difference absolute standard deviation value & DASDV \\
\hline
\end{tabular}

\section{1) Integral of absolute value (IAV)}

IAV is one of the most common sEMG feature and is also called mean absolute value (MAV), average rectified value (ARV), or averaged absolute value (AAV) $[9,22,23]$. IAV is the average of absolute value and can be expressed as follows:

$$
\frac{1}{N} \sum_{i=1}^{N}\left|x_{i}\right|
$$

\section{2) Variance (VAR)}

VAR is often used as a power index of sEMG [9,22,25], and similar with an equation of the variance. VAR is expressed as follows:

$$
\frac{1}{N-1} \sum_{i=1}^{N} x_{i}^{2}
$$

\section{3) Wilson amplitude (WAMP)}

WAMP means the number of times the amplitude of two consecutive signals exceeds a pre-defined threshold (T; in this study $10 \mathrm{mV}$ ) [22]. WAMP is expressed as follows:

$$
\begin{array}{r}
\sum_{i=1}^{N} u\left(\left|x_{i+1}-x_{i}\right|-T\right) \\
\text {,where } \mathrm{u}(\mathrm{x})=1, \text { if } \mathrm{x}>\mathrm{T} \\
\mathrm{u}(\mathrm{x})=0, \text { otherwise }
\end{array}
$$

\section{4) Zero crossing (ZC)}

ZC means the number of times the amplitude of two consecutive signals passes through a value of zero [23,26], and is expressed as follows:

$$
\begin{aligned}
& \sum_{i=1}^{N-1} u\left(-x_{i} x_{i+1}\right) \\
& \text {, where } \mathrm{u}(\mathrm{x})=1, \text { if } \mathrm{x}>0 \\
& \mathrm{u}(\mathrm{x})=0, \text { otherwise }
\end{aligned}
$$




\section{5) Number of turns (NT)}

NT is the number of times the amplitude of three consecutive signals changes from positive to negative [24]. NT is expressed as follows:

$$
\sum_{i=1}^{N-2} u\left[\left(x_{i+1}-x_{i}\right)\left(x_{i+1}-x_{i+2}\right)\right]
$$

\section{6) Mean of amplitude (MA)}

MA means the cumulative change of two consecutive signals, also called waveform length (WL) [23,24], MA is expressed as follows:

$$
\sum_{i=1}^{N-1}\left|x_{i+1}-x_{i}\right|
$$

\section{7) Root mean square (RMS)}

RMS is one of the features commonly used in sEMG signal analysis [21,24], and is expressed as follows:

$$
\sqrt{\frac{1}{N} \sum_{i=1}^{N} x_{i}^{2}}
$$

\section{8) Average amplitude change (AAC)}

AAC is similar with MA feature except averaging [15]. AAC is expressed as follows:

$$
\frac{1}{N} \sum_{i=1}^{N-1}\left|x_{i+1}-x_{i}\right|
$$

\section{9) Difference absolute standard deviation value (DASDV)}

DASDV is similar with RMS and is like a standard deviation of MA mathematically [15,21]. DASDV is expressed as follows:

$$
\sqrt{\frac{1}{N-1} \sum_{i=1}^{N-1}\left(x_{i+1}-x_{i}\right)^{2}}
$$

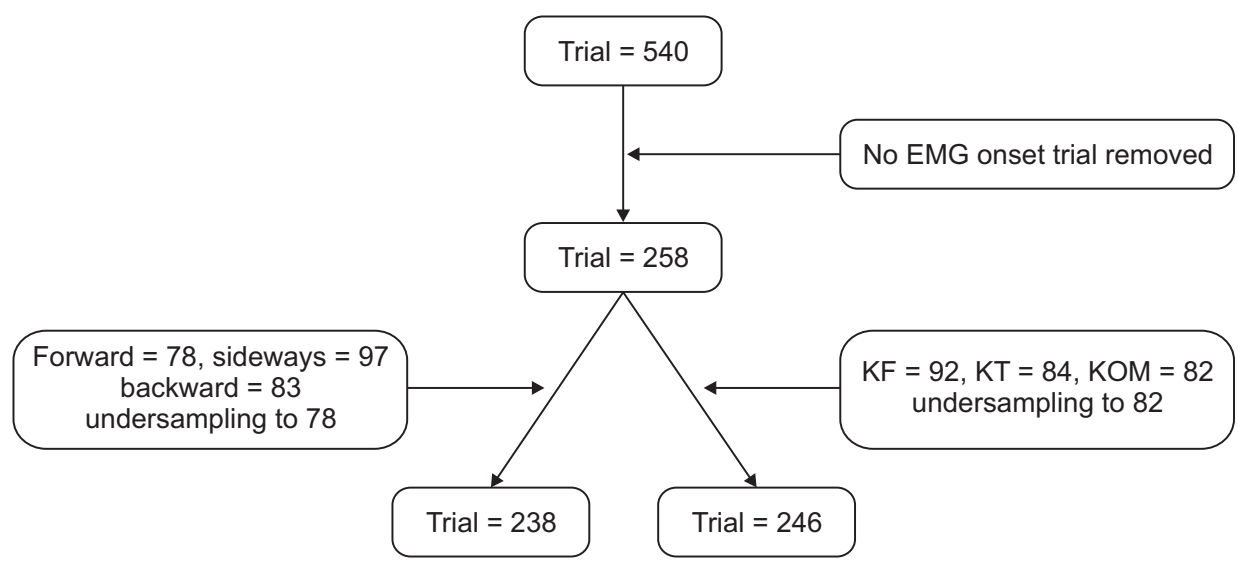

Figure 2. Falling trials included for model construction and evaluation. EMG, electromyography; KF, knee free; $\mathrm{KT}$, knee together; KOM, knee on mat.
A

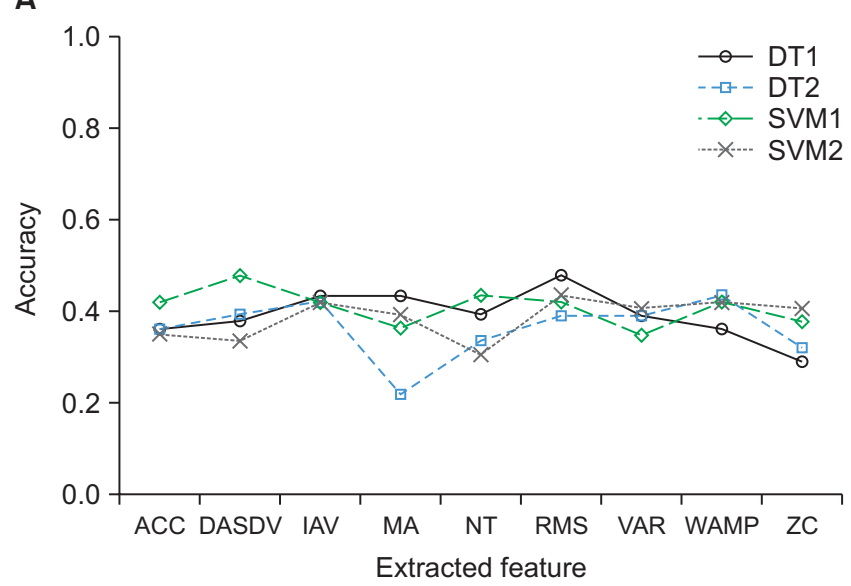

B

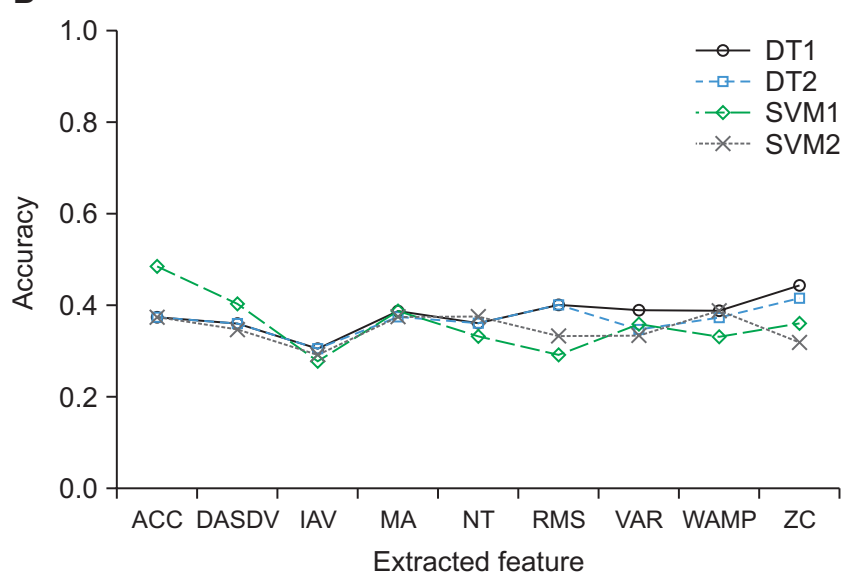

Figure 3. Accuracy of classification model that used various surface electromyography features of hip muscles. (A) Initial fall direction classification. (B) Knee position classification. Accuracy was less than $50 \%$ across all features. DT, decision tree; SVM, support vector machine; SEN, sensitivity; SPE, specificity. 


\section{Classification}

A dataset was divided into $70 \%$ for training and 30\% for testing. The training data were used to construct models and the testing data were used to evaluate the constructed models.

To select models showing optimal performance, two different machine learning techniques with two different parameters ( 4 classifiers) were used. Details on the techniques were provided below.

\section{1) Gaussian kernel support vector machine}

The support vector machine is an algorithm that finds the optimal hyperplane of classification which distinguishes classes of data. "Kernel trick" is a method of classifying what cannot be classified at the original dimension, by interpreting data as a high-level feature space. Support vector machine uses the kernel tricks in different ways (i.e., polynomial kernel, sigmoid kernel, Gaussian kernel), and Gaussian kernel is common due to superior performance. Therefore, Gaussian kernel trick has been used to pattern recognition of human movement [17,29].

The Gaussian kernel support vector machine produces a model by adjusting the cost (c) and gamma $(\gamma)$ as parameters. Cost (c) represents the error tolerance of an outlier, and gamma $(\gamma)$ refers to the range of influence of a single datum, which represents the curvature of the hyperplane. Therefore, the Gaussian kernel support vector machine finds the best performance model by adjusting the parameters. In this study, two models were created by Gaussian kernel support vector machine with the following parameter values:

SVM1: parameter range: $\mathrm{c}=10^{(-5: 1)}, \gamma=10^{(0: 2)}$

SVM2: parameter range: $\mathrm{c}=2^{(-3: 3)}, \gamma=2^{(-3: 3)}$

\section{2) Decision tree}

The decision tree is a classifier used a lot in datamining [30-33]. The decision tree consists of nodes and branches to classify data based on split criteria. Entropy index, Gini index, and chi-square of significance are used for the split criteria of decision tree and entropy and Gini index are commonly used. Based on the pre-determined criteria, machine learning proceeds in the direction of increasing purity and decreasing impurity of variables. In this study, two models were created by

Table 2. Sensitivity and specificity of models classifying initial fall directions

\begin{tabular}{|c|c|c|c|c|c|c|c|c|c|}
\hline \multirow{2}{*}{ Feature } & \multirow{2}{*}{ Class } & \multicolumn{2}{|c|}{ DT1 } & \multicolumn{2}{|c|}{ DT2 } & \multicolumn{2}{|c|}{ SVM1 } & \multicolumn{2}{|c|}{ SVM2 } \\
\hline & & SEN & SPE & SEN & SPE & SEN & SPE & SEN & SPE \\
\hline \multirow[t]{3}{*}{ ACC } & Forward & 0.57 & 0.59 & 0.61 & 0.59 & 0.78 & 0.37 & 0.57 & 0.43 \\
\hline & Sideways & 0.35 & 0.76 & 0.35 & 0.74 & 0.22 & 0.89 & 0.17 & 0.91 \\
\hline & Backward & 0.17 & 0.70 & 0.13 & 0.72 & 0.26 & 0.87 & 0.30 & 0.67 \\
\hline \multirow[t]{3}{*}{ DASDV } & Forward & 0.30 & 0.80 & 0.22 & 0.87 & 0.83 & 0.43 & 0.74 & 0.39 \\
\hline & Sideways & 0.52 & 0.63 & 0.70 & 0.50 & 0.30 & 0.87 & 0.17 & 0.85 \\
\hline & Backward & 0.30 & 0.63 & 0.26 & 0.72 & 0.30 & 0.91 & 0.09 & 0.76 \\
\hline \multirow[t]{3}{*}{ IAV } & Forward & 0.78 & 0.5 & 0.74 & 0.52 & 0.96 & 0.33 & 0.91 & 0.39 \\
\hline & Sideways & 0.17 & 0.98 & 0.17 & 0.98 & 0.17 & 0.93 & 0.17 & 0.89 \\
\hline & Backward & 0.35 & 0.67 & 0.35 & 0.63 & 0.13 & 0.87 & 0.17 & 0.85 \\
\hline \multirow[t]{3}{*}{ MA } & Forward & 0.91 & 0.26 & 0.13 & 0.72 & 0.91 & 0.13 & 0.87 & 0.22 \\
\hline & Sideways & 0.39 & 0.89 & 0.35 & 0.48 & 0.04 & 1 & 0.22 & 0.93 \\
\hline & Backward & 0 & 1 & 0.17 & 0.63 & 0.13 & 0.91 & 0.09 & 0.93 \\
\hline \multirow[t]{3}{*}{ NT } & Forward & 0.43 & 0.59 & 0.30 & 0.70 & 0.48 & 0.48 & 0.30 & 0.72 \\
\hline & Sideways & 0.61 & 0.63 & 0.39 & 0.72 & 0.57 & 0.87 & 0.17 & 0.83 \\
\hline & Backward & 0.13 & 0.87 & 0.30 & 0.59 & 0.26 & 0.80 & 0.43 & 0.41 \\
\hline \multirow[t]{3}{*}{ RMS } & Forward & 0.61 & 0.78 & 0.48 & 0.74 & 0.87 & 0.52 & 0.91 & 0.50 \\
\hline & Sideways & 0.35 & 0.70 & 0.22 & 0.70 & 0.26 & 0.70 & 0.26 & 0.76 \\
\hline & Backward & 0.48 & 0.74 & 0.48 & 0.65 & 0.13 & 0.91 & 0.13 & 0.89 \\
\hline \multirow[t]{3}{*}{ VAR } & Forward & 0.78 & 0.41 & 0.78 & 0.41 & 1 & 0.07 & 1 & 0.13 \\
\hline & Sideways & 0.39 & 0.67 & 0.39 & 0.67 & 0.04 & 0.98 & 0.04 & 1 \\
\hline & Backward & 0 & 1 & 0 & 1 & 0 & 0.98 & 0.17 & 0.98 \\
\hline \multirow[t]{3}{*}{ WAMP } & Forward & 0.43 & 0.57 & 0.91 & 0.33 & 0.35 & 0.74 & 0.43 & 0.74 \\
\hline & Sideways & 0.35 & 0.83 & 0.39 & 0.83 & 0.35 & 0.83 & 0.35 & 0.80 \\
\hline & Backward & 0.30 & 0.65 & 0 & 1 & 0.57 & 0.57 & 0.48 & 0.59 \\
\hline \multirow[t]{3}{*}{ ZC } & Forward & 0.70 & 0.22 & 0.26 & 0.63 & 0.52 & 0.63 & 0.48 & 0.72 \\
\hline & Sideways & 0.13 & 0.76 & 0.35 & 0.61 & 0.43 & 0.72 & 0.39 & 0.67 \\
\hline & Backward & 0.04 & 0.96 & 0.35 & 0.74 & 0.17 & 0.72 & 0.35 & 0.72 \\
\hline
\end{tabular}

DT, decision tree; SVM, support vector machine; SEN, sensitivity; SPE, specificity. 
decision tree:

DT1: entropy index was used.

DT2: Gini index was used.

All model constructions and evaluations were performed with packages (caret, tree, e1071, rpart) provided by RStudio (ver. 1.2.5042; RStudio, Boston, MA, USA).

\section{RESULTS}

\section{Model of Fall Direction}

Total 540 trials were collected in three fall directions. After removing 282 trials that did not show a pre-defined onset time for muscle contraction, 258 trials remained for forward falls (78 trials), sideways falls (97 trials), and backward falls (83 trials). Total 234 trials were selected by under-sampling for model construction (Figure 2).

For the initial fall direction, accuracy of the best model (SVM1 with a DASDV feature) was $48 \%$, and the worst model (DT2 with a MA feature) was 22\% (Figure 3A).

Sensitivity and specificity for each fall direction are summa- rized in Table 2, and there was no model which has sensitivity and specificity of $80 \%$ or greater.

\section{Model of Knee Position}

After removing inappropriate data, 258 falling trials remained for KOM (82 trials), KT (84 trials), and KF (83 trials). Total 246 trials were selected by under-sampling (Figure 2).

For the knee position, accuracy of the best model (SVM1 with an AAC feature) was 49\% and the worst model (SVM1 with an IAV feature) was 29\% (Figure 3B).

Sensitivity and specificity for each knee position during a fall are summarized in Table 3, and there was no model which has sensitivity and specificity of $80 \%$ or greater.

\section{DISCUSSION}

The purpose of this study was to find a best model to classify injurious versus non-injurious falls using sEMG data of hip muscles during a fall, and the injurious falls were defined based on previous studies, where a. the risk of hip fracture increases

Table 3. Sensitivity and specificity of models classifying knee position at the time of hip impact

\begin{tabular}{|c|c|c|c|c|c|c|c|c|c|}
\hline \multirow{2}{*}{ Feature } & \multirow{2}{*}{ Class } & \multicolumn{2}{|c|}{ DT1 } & \multicolumn{2}{|c|}{ DT2 } & \multicolumn{2}{|c|}{ SVM1 } & \multicolumn{2}{|c|}{ SVM2 } \\
\hline & & SEN & SPE & SEN & SPE & SEN & SPE & SEN & SPE \\
\hline \multirow[t]{3}{*}{$\mathrm{ACC}$} & $\mathrm{KF}$ & 0.46 & 0.67 & 0.46 & 0.67 & 0.5 & 0.79 & 0.58 & 0.54 \\
\hline & KT & 0.33 & 0.71 & 0.33 & 0.71 & 0.46 & 0.65 & 0.13 & 0.79 \\
\hline & KOM & 0.33 & 0.69 & 0.33 & 0.69 & 0.5 & 0.79 & 0.42 & 0.73 \\
\hline \multirow[t]{3}{*}{ DASDV } & $\mathrm{KF}$ & 0.63 & 0.40 & 0.75 & 0.23 & 0.58 & 0.5 & 0.54 & 0.46 \\
\hline & $\mathrm{KT}$ & 0.42 & 0.77 & 0.21 & 0.94 & 0.42 & 0.71 & 0.33 & 0.79 \\
\hline & KOM & 0.04 & 0.88 & 0.13 & 0.88 & 0.21 & 0.90 & 0.17 & 0.77 \\
\hline \multirow[t]{3}{*}{ IAV } & $\mathrm{KF}$ & 0 & 1 & 0 & 1 & 0.29 & 0.69 & 0.38 & 0.65 \\
\hline & $\mathrm{KT}$ & 0.33 & 0.60 & 0.33 & 0.60 & 0.33 & 0.56 & 0.29 & 0.69 \\
\hline & KOM & 0.58 & 0.35 & 0.58 & 0.35 & 0.21 & 0.67 & 0.21 & 0.60 \\
\hline \multirow[t]{3}{*}{ MA } & $\mathrm{KF}$ & 0.71 & 0.44 & 0.71 & 0.40 & 0.29 & 0.85 & 0.29 & 0.85 \\
\hline & $\mathrm{KT}$ & 0.42 & 0.67 & 0.42 & 0.67 & 0.38 & 0.67 & 0.38 & 0.67 \\
\hline & KOM & 0.04 & 0.98 & 0 & 1 & 0.5 & 0.56 & 0.5 & 0.56 \\
\hline \multirow[t]{3}{*}{ NT } & $\mathrm{KF}$ & 0.33 & 0.75 & 0.33 & 0.75 & 0.42 & 0.56 & 0.42 & 0.56 \\
\hline & $\mathrm{KT}$ & 0.17 & 0.85 & 0.17 & 0.85 & 0.29 & 0.73 & 0.29 & 0.73 \\
\hline & KOM & 0.58 & 0.44 & 0.58 & 0.44 & 0.29 & 0.71 & 0.29 & 0.71 \\
\hline \multirow[t]{3}{*}{ RMS } & $\mathrm{KF}$ & 0.67 & 0.33 & 0.67 & 0.33 & 0.5 & 0.5 & 0.5 & 0.5 \\
\hline & $\mathrm{KT}$ & 0.38 & 0.92 & 0.38 & 0.92 & 0.29 & 0.69 & 0.29 & 0.69 \\
\hline & KOM & 0.17 & 0.85 & 0.37 & 0.85 & 0.08 & 0.75 & 0.08 & 0.75 \\
\hline \multirow[t]{3}{*}{ VAR } & $\mathrm{KF}$ & 0.67 & 0.27 & 0.25 & 0.73 & 0.42 & 0.60 & 0.42 & 0.60 \\
\hline & $\mathrm{KT}$ & 0.21 & 0.92 & 0.17 & 0.79 & 0.29 & 0.75 & 0.29 & 0.75 \\
\hline & KOM & 0.29 & 0.90 & 0.63 & 0.5 & 0.38 & 0.69 & 0.38 & 0.69 \\
\hline \multirow[t]{3}{*}{ WAMP } & $\mathrm{KF}$ & 0.63 & 0.38 & 0.5 & 0.46 & 0.54 & 0.42 & 0.54 & 0.42 \\
\hline & $\mathrm{KT}$ & 0.25 & 0.90 & 0.42 & 0.77 & 0.25 & 0.79 & 0.25 & 0.79 \\
\hline & KOM & 0.29 & 0.81 & 0.21 & 0.83 & 0.21 & 0.79 & 0.21 & 0.79 \\
\hline \multirow[t]{3}{*}{ ZC } & $\mathrm{KF}$ & 0.29 & 0.88 & 0.25 & 0.81 & 0.33 & 0.71 & 0.33 & 0.71 \\
\hline & $\mathrm{KT}$ & 0.5 & 0.63 & 0.5 & 0.63 & 0.5 & 0.56 & 0.5 & 0.56 \\
\hline & KOM & 0.54 & 0.67 & 0.5 & 0.69 & 0.25 & 0.77 & 0.25 & 0.77 \\
\hline
\end{tabular}

DT, decision tree; SVM, support vector machine; SEN, sensitivity; SPE, specificity; KF, knee free; KT, knee together; KOM, knee on mat. 
10 times when falls initiated backward and landed sideways [7], and b. hip impact force increases 60\% (thereby hip fracture risk increases) when the impacting-side knee is free of constraint ("free knee" - neither the ground nor the contralateral knee contacts the impacting-side knee) at the time of hip impact [6]. We found that classification of initial fall direction through sEMG of hip muscles was not promising, and the accuracy of the best model was less than 50\%. Moreover, classification of knee position at the time of hip impact was not encouraging, either, and the best model provided $49 \%$ of accuracy at most. A potential reason for this poor performance may include target muscles that inappropriate to differentiate injurious versus non-injurious falls. In our experiment, it was difficult to identify EMG onset from the four hip muscles, and considerable number of trials needed to be excluded from data set. However, a previous study that used Gaussian kernel support vector machine showed that a model used sEMG data of the gastrocnemius and tibialis anterior muscles pretty successfully classified activities of daily living versus falls, providing 98.7\% of sensitivity and $98.6 \%$ of specificity [17]. Therefore, future studies should consider other muscle groups (i.e., trunk muscles) along with different data mining techniques to generate the best model for classification of injurious versus noninjurious falls using sEMG data during a fall.

There are limitations in this study. sEMG data we used for model development were acquired when young adults mimicked older adults' falls in laboratory environment. Therefore, our results should be interpreted in light of these limitations.

This is the first study that used data mining techniques to classify injurious versus non-injurious falls using sEMG data of lower extremity muscles during a fall. While classifying models developed in this study showed poor accuracy, the results of this study should provide baseline information, which help guide the design of future study.

\section{CONCLUSIONS}

While it is important to differentiate injurious versus noninjurious falls, limited efforts have been made. To address this issue, we developed classification models based on data mining techniques. However, our results suggest that the classification model built upon the sEMG features of the four hip muscles are not effective to differentiate injurious vs noninjurious falls. Future studies should consider different muscles along with other data mining techniques.

\section{ACKNOWLEDGEMENTS}

This study was supported by the "Brain Korea 21 FOUR Project", the Korean Research Foundation for Department of Physical Therapy in the Graduate School of Yonsei University.

\section{CONFLICTS OF INTEREST}

No potential conflict of interest relevant to this article was reported.

\section{AUTHOR CONTRIBUTIONS}

Conceptualization: KL, WJC. Data curation: KL, WJC. Formal analysis: KL. Funding acquisition: WJC. Investigation: KL, WJC. Software: KL. Supervision: WJC. Validation: KL. Writing original draft: KL. Writing - review \& editing: WJC.

\section{ORCID}

Kitaek Lim, https://orcid.org/0000-0001-5475-3196

\section{REFERENCES}

1. Grisso JA, Schwarz DF, Wishner AR, Weene B, Holmes JH, Sutton RL. Injuries in an elderly inner-city population. J Am Geriatr Soc 1990;38(12):1326-31.

2. Empana JP, Dargent-Molina P, Bréart G; EPIDOS Group. Effect of hip fracture on mortality in elderly women: the EPIDOS prospective study. J Am Geriatr Soc 2004;52(5):685-90.

3. Wolinsky FD, Fitzgerald JF, Stump TE. The effect of hip fracture on mortality, hospitalization, and functional status: a prospective study. Am J Public Health 1997;87(3):398-403.

4. Sattin RW. Falls among older persons: a public health perspective. Annu Rev Public Health 1992;13:489-508.

5. Choi WJ, Cripton PA, Robinovitch SN. Effects of hip abductor muscle forces and knee boundary conditions on femoral neck stresses during simulated falls. Osteoporos Int 2015; 26(1):291-301.

6. Lim KT, Choi WJ. Effect of fall characteristics on the severity of hip impact during a fall on the ground from standing height. Osteoporos Int 2020;31(9):1713-9. 
7. Yang Y, Komisar V, Shishov N, Lo B, Korall AM, Feldman F, et al. The effect of fall biomechanics on risk for hip fracture in older adults: a cohort study of video-captured falls in longterm care. J Bone Miner Res 2020;35(10):1914-22.

8. Aziz O, Musngi M, Park EJ, Mori G, Robinovitch SN. A comparison of accuracy of fall detection algorithms (threshold-based vs. machine learning) using waist-mounted tri-axial accelerometer signals from a comprehensive set of falls and non-fall trials. Med Biol Eng Comput 2017;55(1):45-55.

9. Juan C, Xiang C, Minfen S. A framework for daily activity monitoring and fall detection based on surface electromyography and accelerometer signals. IEEE J Biomed Health Inform 2013;17(1):38-45.

10. Ren L, Peng Y. Research of fall detection and fall prevention technologies: a systematic review. IEEE Access 2019;7: 77702-22.

11. Cheng WC, Jhan DM. Triaxial accelerometer-based fall detection method using a self-constructing cascade-AdaBoost-SVM classifier. IEEE J Biomed Health Inform 2013;17(2):41 1-9.

12. Santos GL, Endo PT, Monteiro KHC, Rocha EDS, Silva I, Lynn T. Accelerometer-based human fall detection using convolutional neural networks. Sensors (Basel) 2019;19(7):1644.

13. Roy SH, Cheng MS, Chang SS, Moore J, De Luca G, Nawab SH, et al. A combined sEMG and accelerometer system for monitoring functional activity in stroke. IEEE Trans Neural Syst Rehabil Eng 2009;17(6):585-94.

14. Adewuyi AA, Hargrove LJ, Kuiken TA. An analysis of intrinsic and extrinsic hand muscle EMG for improved pattern recognition control. IEEE Trans Neural Syst Rehabil Eng 2016; 24(4):485-94

15. Phinyomark A, Phukpattaranont P, Limsakul C. Feature reduction and selection for EMG signal classification. Expert Systems with Applications 2012;39(8):7420-31.

16. Sutherland DH. The evolution of clinical gait analysis part $\mathrm{l}$ : kinesiological EMG. Gait Posture 2001;14(1):61-70.

17. Xi X, Tang M, Miran SM, Luo Z. Evaluation of feature extraction and recognition for activity monitoring and fall detection based on wearable sEMG sensors. Sensors (Basel) 2017;17(6): 1229.

18. Criswell E, Cram JR. Cram's introduction to surface electromyography. 2nd ed. Sudbury: Jones and Bartlett; 2011;xxi, 412.

19. Nazmi N, Abdul Rahman MA, Yamamoto S, Ahmad SA, Zamzuri $\mathrm{H}$, Mazlan SA. A review of classification techniques of EMG signals during isotonic and isometric contractions. Sensors
(Basel) 2016;16(8):1304.

20. Englehart K, Hudgins B, Parker PA, Stevenson M. Classification of the myoelectric signal using time-frequency based representations. Med Eng Phys 1999;21(6-7):431-8.

21. Kim KS, Choi HH, Moon CS, Mun CW. Comparison of $k$-nearest neighbor, quadratic discriminant and linear discriminant analysis in classification of electromyogram signals based on the wrist-motion directions. Curr Appl Phys 2011;11(3):7405.

22. Zardoshti-Kermani M, Wheeler BC, Badie K, Hashemi RM. EMG feature evaluation for movement control of upper extremity prostheses. IEEE Trans Rehabil Eng 1995;3(4):324-33.

23. Hudgins B, Parker P, Scott RN. A new strategy for multifunction myoelectric control. IEEE Trans Biomed Eng 1993;40(1): 82-94.

24. Boostani R, Moradi MH. Evaluation of the forearm EMG signal features for the control of a prosthetic hand. Physiol Meas 2003;24(2):309-19.

25. Park SH, Lee SP. EMG pattern recognition based on artificial intelligence techniques. IEEE Trans Rehabil Eng 1998;6(4): 400-5.

26. Chang GC, Kang WJ, Luh JJ, Cheng CK, Lai JS, Chen JJ, et al. Real-time implementation of electromyogram pattern recognition as a control command of man-machine interface. Med Eng Phys 1996;18(7):529-37.

27. Keleş S, Subaşı A. Classification of EMG signals using decision tree methods. Paper presented at: 3rd International Symposium on Sustainable Development (ISSD); 2012 May 31-Jun 1; Sarajevo, Bosnia and Herzegovina. Sarajevo: International Burch University, 2012. p. 354-66.

28. Lee KJ, Lim K, Choi WJ. The effect on the hip muscle activation of the fall direction and knee position during a fall. Phys Ther Korea 2021;28(1):84-91.

29. Wei W, Jia Q. Weighted feature Gaussian kernel SVM for emotion recognition. Comput Intell Neurosci 2016;2016: 7696035.

30. Song W, Han Q, Lin Z, Yan N, Luo D, Liao Y, et al. Design of a flexible wearable smart sEMG recorder integrated gradient boosting decision tree based hand gesture recognition. IEEE Trans Biomed Circuits Syst 2019;13(6):1563-74.

31. Trevisan C, Di Gregorio P, Debiasi E, Pedrotti M, La Guardia M, Manzato E, et al. Decision tree for ward admissions of older patients at the emergency department after a fall. Geriatr Gerontol Int 2018;18(9):1388-92. 
32. Cai WY, Guo JH, Zhang MY, Ruan ZX, Zheng XC, Lv SS. GBDTbased fall detection with comprehensive data from posture sensor and human skeleton extraction. J Healthc Eng 2020; 2020:8887340.
33. Waris A, Niazi IK, Jamil M, Englehart K, Jensen W, Kamavuako

EN. Multiday evaluation of techniques for EMG-based classification of hand motions. IEEE J Biomed Health Inform 2019; 23(4):1526-34. 